\title{
Nature and diagnosis of food allergy
}

\author{
By A. M. Denman, Division of Rheumatology, Clinical Research Centre, Watford \\ Road, Harrow, Middlesex $H_{1}{ }_{3} U \mathcal{J}$
}

\section{Introduction}

The subject of food allergy has enjoyed varying fortunes. This diagnosis was commonly invoked to explain a variety of diseases but usually on slender evidence and with the introduction of systematic, physiological observations into medicine the subject fell into disrepute. However, the discovery of gluten hypersensitivity as the cause of coeliac disease rekindled interest because this represented a disease in which the offending food had been precisely defined. Moreover the toxic substance thought to provoke the disease has been defined in precise chemical terms. The introduction of safe, reproducible techniques for jejunal biopsy and accurate immunological methods, also made it possible to analyse the possible mechanisms by which gluten induces the clinical features of the disease, the histopathological abnormalities in the small intestine, and the systemic complications which commonly accompany this disorder. Such observations raised hopes that other relatively simple foods would account for a variety of gastrointestinal complaints and indeed other disorders. This concept has been most attractive in relation to chronic inflammatory disorders of the gastrointestinal tract such as Crohn's Disease and ulcerative colitis. Not only are the pathological features of these diseases plausibly explained by a persistent reaction to food antigens, but in addition a wide range of serum antibodies against ingested proteins can readily be detected. In addition to such obvious spheres in which to pursue the theme of food allergy, in some susceptible individuals hypersensitivity to some foods such as milk has been shown to produce a disease picture of surprising complexity and moreover of a nature which would not at first sight appear explicable on these grounds. The additional discovery that gluten hypersensitivity may present clinically not as a disease affecting primarily the gastrointestinal tract but predominantly as a skin disorder, dermatitis herpetiformis, has made clinicians once more receptive to the idea that food allergy may cause a wide and protean range of complaints.

The newly aroused enthusiasm for this subject has prompted more speculative suggestions that food allergy can explain a wide range of human afflictions both organic and psychiatric (Mackarness, 1976). Furthermore, there has been increasing interest in the possibility that many chronic inflammatory diseases in advanced societies are related to changing life style. The epidemiological evidence in favour of this view comes largely from comparing the incidence of such disorders in relatively undeveloped areas of Africa and Asia and the frequency of these same diseases in developed societies. There is good evidence that many auto- 
immune and connective tissue diseases are found in greater frequency in developed communities. Of particular interest are studies which have shown that the incidence of such diseases is higher in black South Africans who have migrated to the cities than in people of the same race who have remained in black reservations. Such differences have been attributed to changes in diet. It is important therefore that food allergy be properly defined and that diagnostic criteria combining both clinical and laboratory observations be defined in this as in any other area of clinical practice.

\section{Definition of food allergy}

Food allergy can be defined as hypersensitivity to constituents of food mediated by one or more forms of immunological reaction. There is an important distinction to be made between food allergy and hypersensitivity or intolerance to diet induced by other mechanisms such as, for example, lactose deficiency.

\section{The clinical presentation of food allergy}

It is essential to define reasonable criteria for accepting that a particular clinical disorder may, at least in some patients, be attributed to food allergy. In making a judgement of this kind two points must be considered. Firstly, food allergy must not be diagnosed merely on the grounds that all other reasonable organic causes have been excluded; the diagnosis must be made in a positive manner. Secondly, there are chronic diseases such as multiple sclerosis and schizophrenia in which food allergy has been alleged to play a part. Whilst these theories have interesting research implications such diseases do not come within the scope of routine clinical diagnosis and are not further considered. The conditions on which it is reasonable to consider the possibility of food allergy are shown in Table $\mathrm{I}$. There are two further points to be made about the disorders listed in this Table. Firstly, it is not immediately apparent that many of the organs involved can plausibly be affected by an allergen absorbed through the gut. Whereas it is obvious to think of this aetiology in a patient with gastrointestinal symptoms, it is less so in individuals with, for example, atopic eczema. Secondly, many common disease features have more than one cause and it is only practicable to establish a dietary aetiology when other recognized causes have been excluded. For example, recurrent aphthous ulcers may result from simple infections, drug hypersensitivity, or accompany systemic diseases; only after causes of this kind have been eliminated can recourse be made to exclusion diets. Nonetheless it should be recognized that food allergy may provoke disease manifestations of an unusual or even bizarre nature including psychiatric symptoms, recurrent aphthous ulcers, and abnormalities of heart rate (Finn \& Cohen, 1978). Similarly allergy to a variety of food allergens has been implicated in the pathogenesis of an uncommon hypersensitivity disorder, eosinophilic gastroenteritis (Caldwell et al. 1975) although this is not the basis of the disease in all patients (Klein et al. 1970). Similarly hypersensitivity to cow's milk may precipitate pulmonary haemosiderosis leading to heart disease in young children (Boat et al. 1975). 
Table I. Clinical manifestations of food allergy

$\begin{array}{lll}\text { System affected } & \text { Principal disorders } & \text { Allergen } \\ \text { Gastrointestinal } & \text { Coeliac disease } & \text { Gluten } \\ & \text { Granulomatous enteritis } & \text { Milk } \\ & \begin{array}{l}\text { Abdominal pain, diarrhoea } \\ \text { Aphthous ulcers }\end{array} & \text { Various } \\ & \text { Various } \\ \text { Skin } & \text { Dermatitis herpetiformis } & \text { Gluten } \\ & \text { Urticaria } & \text { Various } \\ \text { Atopic eczema } & \text { Various } \\ \text { Respiratory } & \text { Extrinsic asthma } & \text { Various } \\ & \begin{array}{l}\text { Allergic rhinitis } \\ \text { Pulmonary haemasiderosis }\end{array} & \text { Various } \\ \text { Central nervous system } & \text { Migraine } & \text { Chocolate \& others } \\ & \text { Occasional behaviour } & \\ & \text { problems } & \text { Various }\end{array}$

\section{The pathogenesis of food allergy}

Earlier oversimplified views about the pathogenesis of food allergy have needed extensive modification following recent discoveries. It used to be thought that dietary proteins or other potential allergens were not normally absorbed in a form which could precipitate an immune response. Thus it was assumed that the detection of precipitating antibodies to food proteins in the serum of patients with alleged food allergy was a pathological finding. It is now recognized that dietary proteins are absorbed both in experimental animals and in man in a form and in quantities sufficient to stimulate an immune response so that circulating antibodies are a physiological response to absorbed antigens and do not have necessarily any pathological connotation. Thus in seeking mechanisms by which absorbed food proteins might provoke an immune response with pathological consequences, one is forced to look for qualitative or quantitative changes in a physiological process which might produce deleterious effects. There are many mechanisms which limit the absorption of antigen from the gut. Some of these are non-immunological and are produced by enzymes such as pancreatic proteases which degrade dietary proteins (Walker et al. 1975). In addition antibody, and more specifically IgA secreted into the lumen of the gastro-intestinal tract, also limits the absorption of proteins in immunogenic form. This antibody has a dual role, since not only does it bind dietary antigens but it also forms complexes with these antigens leading to their absorption to the mucosal surface thereby facilitating enzymatic degradation (Walker et al. 1975). The importance of IgA in blocking antigen absorption has been stressed by Soothill ( 1976 ), who has speculated that early exposure to dietary allergens such as those in cow's milk at a stage when there is relative deficiency of IgA may allow the excessive absorption of such proteins thereby predisposing to excessive antibody formation and hypersensitivity reactions. Certainly the capacity to form specific antibody to ingested food antigens is acquired at an early age and antibodies to cow's milk protein have been detected in premature infants born after $3^{6}$ to $3^{8}$ weeks gestation (Rieger \& Rothberg, 1975). The importance of limiting 


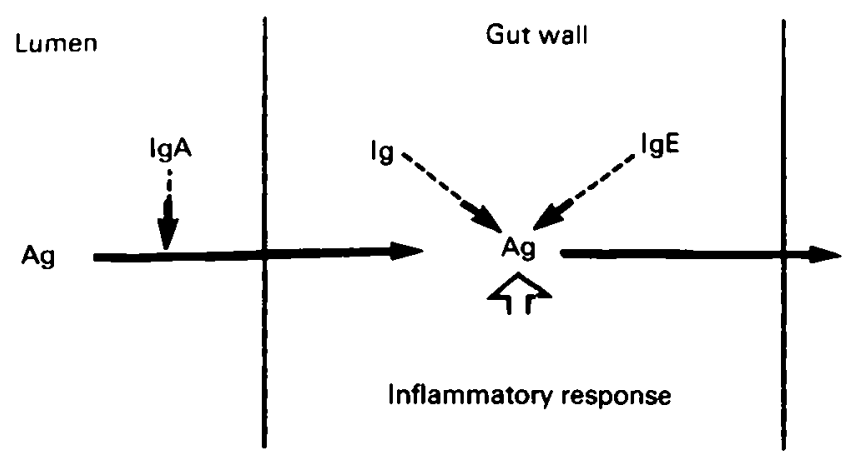

Blood

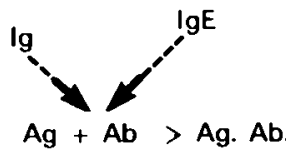

Fig. 1. The pathogenesis of food allergy. The rate of antigenic ( $\mathrm{Ag})$ absorption is controlled by scveral factors including immunoglobulin $A(\operatorname{IgA})$. In the gut wall the combination of antigen with immunoglobulin $\mathrm{G}, \mathrm{M}(\mathrm{Ig})$ or immunoglobulin $\mathrm{E}$ (IgE) may induce an inflammatory response under certain circumstances. Similarly the combination of antigen with antibody $(\mathrm{Ab})$ of any class may lead to the formation of circulating soluble complexes able to induce an inflammatory response (depicted as $\mathrm{Ag}+\mathrm{Ab} \rightarrow \mathrm{Ag} . \mathrm{Ab}$ ). However, the absorption of antigen and the antibody response this invokes does not produce disease in normal individuals.

the absorption of antigen may explain the efficacy of disodium cromoglycate in treating some patients with proven food allergy since this drug has been shown to limit the absorption of egg albumen in patients with atopic symptoms induced by this allergen (Dannaeus \& Johansson, 1979).

Although mechanisms of this kind may limit the extent of antigen absorption and therefore the amount of antibody synthesis this provokes, circulating antibodies are found in the serum of normal individuals lacking any clinical evidence of food allergy. Thus other factors must determine why the clinical features of allergy appear only in susceptible individuals. This susceptibility has been variously attributed to differences in the class of antibody or other characteristics of the invoked antibody response. In other forms of allergy such as allergic rhinitis provoked by sensitivity to grass pollen, symptoms can be attributed to the propensity of atopic individuals to synthesize reaginic antibody of the IgE class. This antibody binds to mast cells and basophils and on exposure to allergens such as those in grass pollen these allergens combine with $\operatorname{IgE}$ passively bound on the surface of these cells thereby triggering the release of histamine and other mediators of acute hypersensitivity reactions (reviewed by Platts-Mills \& Denman, I976).

Another possibility is that dietary antigens may combine with antibody to form soluble complexes in the plasma which circulate for abnormally long periods in this form and are deposited in the mucous membrane of the gastrointestinal tract or in other tissues in which allergic reactions are provoked (Fig. 2). After the absorption of antigen in a normal individual with circulating antibodies to that antigen provoked by previous exposure there is always a brief period during which antigen and antibody circulate as immune complexes before these are cleared from the 


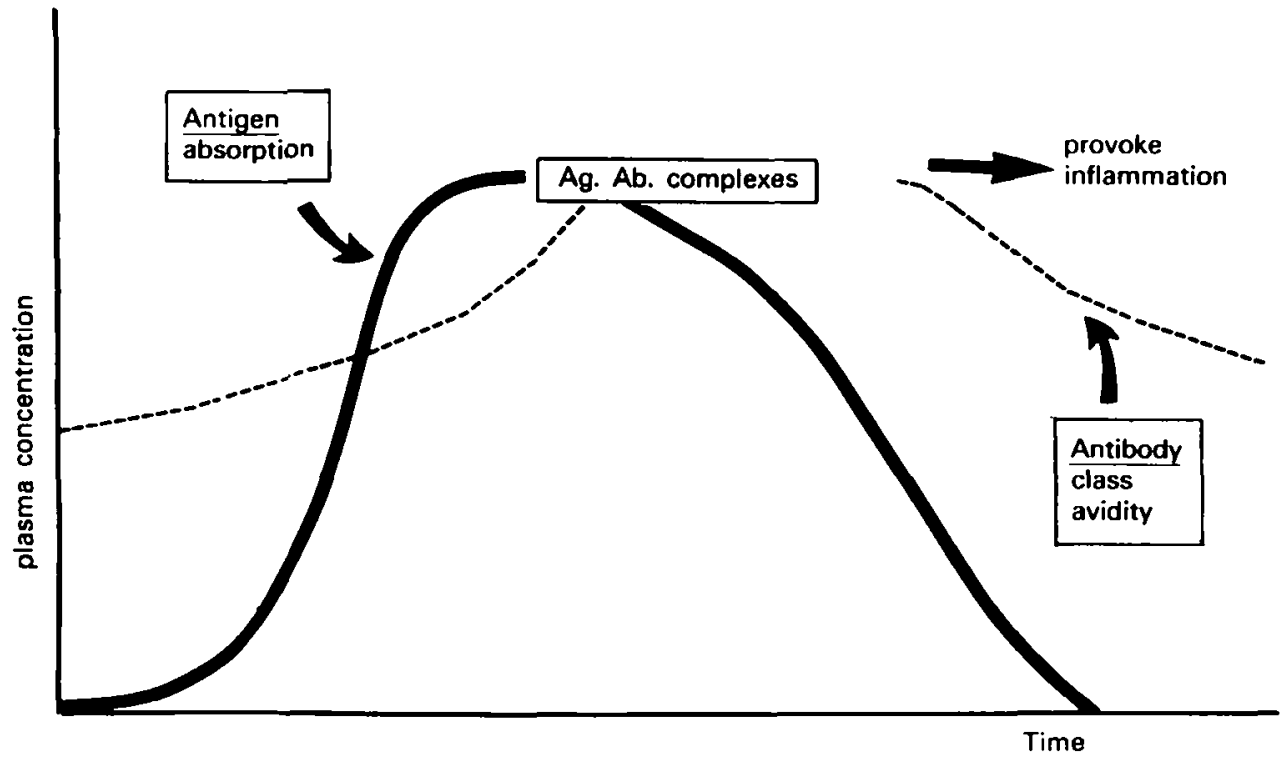

Fig. 2. A schematic representation of the pathogenesis of immune complex disease. As the concentration of dietary antigens rises after meals $\left(-{ }_{-}\right)$these combine with antibody already circulating in the blood (- - ) to form antigen-antibody (Ag.Ab) complexes. This is a fleeting, harmless event in normal individuals since there is an excess of antibody and both antigens and complexes are readily cleared from the circulation. However, if antibody production is impaired, (antibody of certain classes, poor antigen-binding i.e. low avidity) the period during which immune complexes circulate is prolonged and these may provoke an inflammatory reaction. Impaired function of the reticulo-endothelial system (not depicted) has the same effect since Ag.Ab complexes are cleared abnormally slowly.

circulation. Indeed, provided sufficiently sensitive techniques are used, circulating antigen-antibody complexes can be detected after meals in most people. Thus it has been reported that the serum of all human infants fed on cow's milk contains circulating immune complexes consisting of cow's milk proteins as the antigens and IgG of maternal origin (Delire et al. 1978). However, should the period during which antigen-antibody complexes circulate be prolonged, these may be deposited in the tissues and there provoke an inflammatory response by a variety of mechanisms. It has been reported that abnormally high levels of complexes containing food proteins and antibody can be detected in the serum of patients with allergic symptoms. After eating eggs two adult patients with a history of eczema and asthma who were known to be sensitive to egg developed the typical symptoms of their allergy and circulating immune complexes were detected in their plasma (Paganelli et al. 1979). There are several possible explanations for the prolonged circulation of immune complexes in high concentration containing food allergens. Excessive absorption of antigen prolongs the period in which complexes circulate. Antibody of poor avidity is incapable of forming an antigen-antibody lattice of high molecular weight which is readily phagocytosed by the reticuloendothelial system. Finally, defects in the reticulo-endothelial system delay the clearance of immune complexes. 


\section{The diagnosis of food allergy}

Clinical asessment. Careful history-taking and an awareness of the protean manifestations of food allergy remain the most important part of diagnosing food allergy. Other atopic illnesses which the patient may have suffered should be considered since conditions such as bronchial asthma and eczema are very common in patients with food allergy and, for example, 85 out of roo patients with intolerance to specific foods were in one series found to suffer such associated disorders (Lessof et al. 1979). The recognition that food allergy may produce symptoms of common disorders in some patients as well as bizarre symptoms in others should be tempered by the realization that complete objectivity is indispensable. The clinician must collaborate with the responsible dietitian in planning the investigation of individual patients. In the past, it has been customary to devise a series of rotating diets so that potential food allergens can be identified whilst the patient records and compares his symptoms during different phases of the exercise. However, we have found it more convenient to adopt a more stringent diet from the start. In the majority of patients and particularly in those where it is undesirable or impracticable to arrange hospital admission, an exclusion diet can be devised which allows the patient to pursue his normal occupation and activities (Table 2). This diet excludes most potential allergens but it has to be recalled that some fruits provoke urticaria in susceptible individuals and that some patients may react to tea and coffee although not through a mechanism involving true allergy. Where the symptoms are more pressing and the social circumstances allow, it is expedient to admit the patient to hospital to receive fluids and an elemental diet in the form of 'vivonex' only. For most routine clinical purposes the patient can be challenged by systematically reintroducing items of diets omitted in the basic exclusion diet. This is determined by careful history-taking to get some idea of likely allergens. Food items should be replaced sequentially at the rate of one major item/week. In most individuals, in whom the clinical manifestations such as urticaria are obvious, there is no need to record the response to challenge under controlled double-blind conditions. It is sufficient to ask the patient to keep a careful diary recording his symptoms on a standard scale of severity. However, in patients with unusual symptoms where the diagnosis may be suspected but is not clear-cut, it is essential that the series of provocation tests be given in a controlled, double-blind manner. After a basic period on an exclusion diet the

\section{Table 2. Basic exclusion diet: principal features}

Permitted

Fresh fruit and vegetables

Fresh fruit juice

Tea, coffee, sugar

Rice

Gluten-free bread

Olive oil

Barley sugar

Tomor margarine
Major exclusions

Meat and poultry

Eggs

Milk and dairy products

Tinned, frozen and preserved food 
patient is challenged with a series of suspected allergens or control placebos. Challenge and placebo materials are matched for taste and consistency and are given in quantities of $3^{\circ} \mathrm{g}$ at $2 \mathrm{~d}$ intervals. Ideally these should be made up by one dietitian who devises the sequence of testing according to a pre-arranged code and the substances should then be administered by a second dietitian. During each period of challenge, the patient records his response on standard sheets or occasionally this is assessed on a visual analogue scale. In many patients an exclusion diet is combined with the regular administration of disodium cromoglycate ('Nalcrom', Fisons Pharmaceuticals) so as to permit a more liberal diet. It is essential that the true value of this drug in individual patients be tested by determining the extent to which disodium cromoglycate or an identical placebo suppresses the symptoms provoked by a known allergen.

\section{Laboratory investigations and food allergy}

As with other forms of hypersensitivity, reactions to food allergens fall into two principal categories namely immediate responses mediated by serum antibody and delayed responses mediated by various lymphocyte populations. Different kinds of laboratory test are used to detect these responses. Such tests can again be considered in two categories, firstly those involving a study of in vivo reactions and secondly in vitro tests for humoral or cell-mediated immune reactions to suspected allergens. Skin tests are the simplest in vivo test for immediate hypersensitivity and such 'prick' tests are positive in a high percentage of patients with food allergy (Galant et al. 1973). However, the results are often non-specific and it is not often clear whether these reflect specific food allergy or the generally atopic condition of many of the individuals being tested. Nevertheless the combination of a strongly positive skin test, and a high specific IgG antibody level may be diagnostic, as for example in patients with egg allergy (Davies \& Pepys, 1976). It is also possible to look for an immediate sensitivity reaction at the site of exposure to antigen by performing jejunal biopsies. There have been claims that it is possible to discern specific changes of this kind either in the form of an infiltration of mast cells and basophils or by detecting IgE in the lesion. However, there are doubts about the specificity of such tests. In vitro tests involve the demonstration of sensitized basophils or of antibody of a class or in a form likely to be associated with an immediate hypersensitivity reaction. The most important of these tests are those which purport to detect IgE specifically reactive with the suspected allergen. The commonly used techniques for this purpose are various forms of radioimmunoassay. Some investigators use tests which provoke the release of mediators from basophils allergized by IgE. A high percentage of positive tests for specific IgE have been reported in patients with food allergies (Galant et al. 1973; Hoffman \& Haddad, 1974; Chua et al. 1976). However, many of these assays are of uncertain reliability. As already stressed, the detection of antibody of other classes is not necessarily of pathological significance. Although there are numerous assays for detecting circulating immune complexes in plasma, it can not be assumed that these contribute to the pathogenesis of the disease or are of 
diagnostic importance. Moreover a wide range of tests is necessary in order to detect immune complexes in all forms in which these may circulate whilst false positive results are common. Nevertheless the detection of immune complexes composed of suspected allergens in combination with IgE or of high molecular weight may prove to have diagnostic significance (Brostoff et al. 1979, Paganelli et al. 1979). More recently attention has been given to the possibility that biopsies obtained from the gastro-intestinal tract can be induced to synthesize specific antibody in vitro but this technique is not yet routinely available.

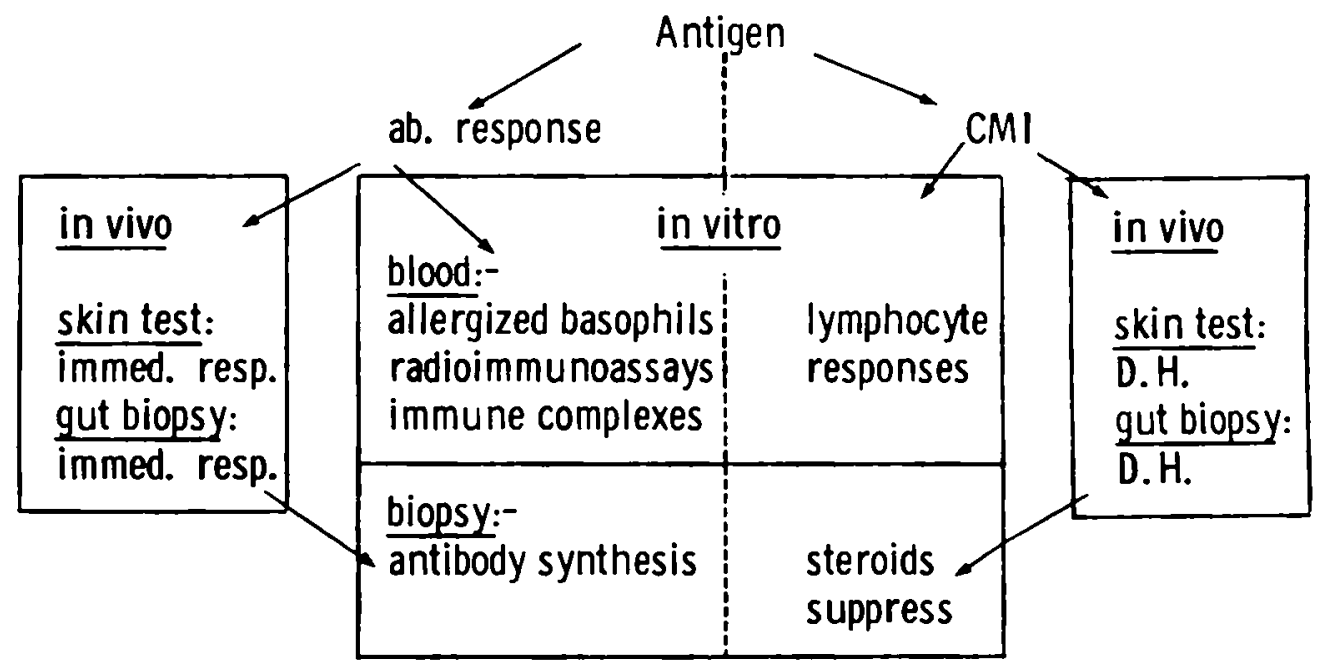

Fig. 3. Immunological tests for food allergy. Antigen invokes an antibody (ab) response or cellmediated immunity (CMI). The ab response can be detected by in vivo tests so that after oral challenge the gut can be examined for evidence of an antibody response (immed. resp.) or skin tests can be undertaken for the same purpose. A variety of in vitro tests indicated in the text are available for detecting antibody responses using blood samples or jejunal biopsies.

CMI can also be detected in vivo but the skin and gut show delayed hypersensitivity (DH) responses characteristic of this form of immunity. Blood cells and jejunal biopsies can also be adapted for in vitro tests of CMI.

There are also in vivo tests available for the detection of delayed hypersensitivity reactions to suspected food allergens. Skin tests are the most convenient to employ and involve the intradermal injection of purified allergen. These reactions can be distinguished from those of immediate type by their appearance only after an interval of 24 to $48 \mathrm{~h}$ and by the histological appearances of the reaction. This technique has been applied to the diagnosis of suspected gluten hypersensitivity. However, it must be pointed out firstly that the distinction between immediate and delayed type hypersensitivity reactions is not absolute and secondly, that it is also common to obtain false positive reactions in patients with atopic diseases. In contrast to immediate hypersensitivity reactions, the histological changes in jejunal biopsies are often more specific particularly in the context of gluten hypersensitivity. It is now possible to quantitate the degree of lymphocytic infiltration in jejunal biopsies in an objective manner. In contrast there are few in vitro tests for delayed hypersensitivity to food allergens which 
have proved simple or reliable. In theory, and in some contexts of clinical practice, lymphocytes sensitized to specific allergens are activated in vitro by exposure to these allergens. Unfortunately lymphocyte 'transformation', as it is termed, has not proved useful in the diagnosis of food allergy even when the basic test has been considerably modified. Techniques are also being devised whereby organ cultures established from jejunal biopsies can be exposed to suspected allergens and examined for signs of specific damage. For example the cultured jejunal mucosa of patients with gluten hypersensitivity shows biochemical evidence of damage after exposure to gluten in vitro and these abnormalities can be reversed by cortisone (Katz et al. 1976).

\section{Specific examples of food allergy}

It is appropriate at this point to give some specific examples of how a combination of clinical examination and laboratory tests may be used to establish the diagnosis of food allergy and, what is sometimes equally important, to refute it when necessary. Urticaria is one condition in which food allergy plays a part in some patients. This is a disease characterized by an itchy rash occasionally accompanied by oedema of mucous membranes so that the tongue and pharynx may also be affected. The disease can give considerable symptomatic distress and may persist for a period of months or years. In common with the experience of others we found that $40 \%$ of patients presenting with chronic urticaria responded to an exclusion diet (Denman et al. 1976). The provoking substances most commonly encountered were dairy products, eggs, and foods containing salicylates or benzoic acid derivatives. There are two points about such patients which illustrate the difficulties of making a firm diagnosis of food allergy. Firstly remission may be incomplete so that the patients will tolerate their disorder more easily on a modified diet but they do not always enjoy a complete remission. This probably means that other factors contribute to the urticaria. The variable natural history of chronic urticaria and the observation that salicylates provoke exacerbation of urticaria in most patients with this disorder irrespective of the cause (Doeglas, 1975) emphasize this point. Secondly it is not established that foods which provoke urticaria always do so by an immunological mechanism. Many patients have specifically reactive $\operatorname{lgE}$ and other serological changes which indicate that an immunological mechanism may be responsible but this is not always the case (Denman et al. 1976).

Usually the diagnosis of food allergy is less obvious and the clinical investigation has to be more methodical as the following case reports illustrate. A I 5 year old schoolgirl presented with a history of recurrent swelling of the lymph nodes in the neck and nervous symptoms which took the form of hearing noises, irrational fears which made her hide in her bedroom for long intervals, bouts of depression, and insomnia. At the age of four she had suffered recurrent abdominal pain which had responded to a milk exclusion diet. She was given a series of challenges with milk or a matched placebo either alone or in combination with disodium cromoglycate. 
Symptoms were recorded by the patient, by her physicians, and by a psychiatrist, and blood was taken at regular intervals to seek evidence of an immunological reaction. Only after exposure to milk did she develop symptoms and a raised IgE in combination with splitting of $\mathrm{C}_{3}$ reflecting complement activation. This patient therefore was diagnosed by clinical and laboratory criteria.

On occasion the laboratory findings are not helpful. A $5^{\circ}$ year old lady presented with a 7 year old history of violent abdominal pain, palpitations, generalized flushing with fever, followed by several days of intractable diarrhoca. She had devised an exclusion diet since she was convinced that her attacks were precipitated by meat, fish, eggs, some varieties of cheese, green vegetables, dried fruit, chocolate, and alcohol. She was married to a Garden Superintendent and had orginally two children but one son had died at the age of 27 from a perforated duodenal ulcer. This had aroused the suspicion that her symptoms were psychosomatic in origin. However, there was a strong family history of atopic disorders and the patient herself was known to be sensitive to penicillin. Our psychiatric assessment did not reveal any abnormality. Accordingly she was put on an exclusion diet and given a series of coded challenges interspersed with placebos. The patient at no time developed evidence of an immunological reaction judged from serial examination of her serum and from sequential jejunal biopsies. Nonetheless her own assessment of her symptoms and the judgement of the clinical observers showed that her symptoms were provoked only by specific antigens. In contrast to these two patients, objective testing may be necessary to refute an alleged diagnosis of food allergy. A 53 year old lady presented with a history of several years of uncontrollable weepiness, disorientation, and abdominal pain. She had become virtually a social recluse and her illness was provoking grave repercussions in her family. She had been given a series of sub-lingual challenges with various foodstuffs and was told that she was suffering from 'masked food allergy' on the basis of these results. She had been put on a diet of 'vivonex' and tapioca and as a result lost 15 kilograms of weight in a few weeks. She was admitted to hospital and maintained on a diet consisting exclusively of 'vivonex'. She was given a series of coded challenges containing alleged allergens or placebo at $48 \mathrm{~h}$ intervals taking precautions that she could not identify these by taste, appearance or consistency. The details of her clinical assessment are given in Table 3 . Her symptoms were unchanged by this sequence of challenges and no evidence was obtained that she

\section{Table 3. Double-blind assessment of patient with alleged 'masked allergy'}

(I.aboratory samples and ECG tapes were coded before analysis)

1. Clinical assessment (medical and psychiatric)

2. Self assessment (visual analogue scales)

3. Continuous $24 \mathrm{~h}$ electrocardiographic (ECG) recordings

4. Immunological tests - immunoglobulins in IgE circulating immune complexes complement activation 
was suffering from food allergy. She has been restored to a normal diet and on this and with orthodox psychiatric help she is making considerable improvement.

\section{Conclusions}

There is no doubt that true allergy to various food allergens accounts for organic disease in many patients. Not only should this diagnosis be considered in patients with symptoms which obviously could be related to this cause, such as urticaria, recurrent aphthous ulcers, and unexplained abdominal pain and discomfort but it is an occasional cause of diseases where the link with food allergy may not be immediately apparent. The importance of considering this diagnosis is now widely accepted (May \& Block, 1978; Zanjanian, 1976). It is also widely appreciated that clinical assessment and particularly the response to exclusion diets followed by challenge with selected allergens is the surest way of making the diagnosis. Laboratory tests are becoming increasingly helpful and more specific but cannot be relied upon in isolation. The importance of double-blind control studies in attributing the symptoms of individual patients or patients with common conditions to food allergy is also well accepted. For example the importance of avoiding dietary antigens in atopic eczema has been shown in a proper doubleblind controlled trial (Atherton et al. 1978). This principle is also well established in paediatric practice (Johnstone, I978).

Therapeutic trials of drugs such as disodium cromoglycate must also be done in a controlled fashion. For example, there are many uncontrolled studies showing that this drug benefits patients with food allergy (Esteban et al. 1977; Frier \& Berger, 1973; Dahl, 1978), but few reports of controlled trials in food allergy. For example, despite reports to the contrary, we found in a double-blind controlled trial that this drug did not benefit patients with chronic urticaria (Denman et al. 1979). It is essential that proper studies of this kind be carried out if an important subject is not to fall into disrepute. Thus sweeping condemnations have been made of 'food additives' largely on emotional grounds, overlooking the fact that without advances in the technology of handling foods under-nutrition would be the inevitable consequence. Whilst some individuals may show true hypersensitivity to various preservatives and other materials added to food, this issue also needs objective assessment (Jukes, 1977). A similarly critical attitude is needed in assessing the results of laboratory studies implicating food allergy on the basis of serological tests. Whilst antibodies against dietary proteins may be found in higher titres in the blood of patients with inflammatory bowel disease than in blood from normal controls, it is likely that these antibodies are the result and not the cause of the inflammatory process in the bowel wall (Falchuk \& Isselbacher, 1976).

The author is grateful to Miss P. J. Brereton and her colleagues in the Department of Dietetics for their continued help with these studies; he is also grateful to Mrs Valerie Hartman for help in preparing the manuscript. All clinical studies referred to in this paper were approved by the Ethical Committee of Northwick Park Hospital. 
Atherton, D. J., Sewell, M., Soothill, J. H., Wells, R. S. \& Chilvers, C. E. D. (1978). Lancet i, 401. Boat, T. F., Polmar, S. H., Whitman, V., Kleinerman, J. I., Stern, R. C. \& Doershuk, C. F. (I975). f. Pediat. 87, 23.

Brostoff, J., Carini, C., Wraith, D. G. \& Johns, P. (1979). Lancet i, 1268.

Caldwell, J. H., Tennenbaum, J. I. \& Bronstein, H. A. (1975). New Eng. F. Med. 292, 1388.

Chua, Y. Y., Bremner, K., Lakdawalla, N., Llobet, J. L., Kokubu, H. L., Orange, R. P. \& CollinsWilliams, C. (1976). J. Allergy clin. Immunol. 58, 299.

Dahl, R. (1978). Allergy 33, 120.

Dannaeus, A. \& Johansson, S. G. O. (1979). Proc. Int. Mtg Mast Cell (In the Press).

Davies, R. \& Pepys, J. (1976). J. Allergy clin. Immunol. 57, 373.

Delire, M., Cambiaso, C. L. \& Masson, P. L. (1978). Nature, Lond. 272, 632.

Denman, A. M., Kingsley, P. J. \& Brereton, P. J. (1979). Proc. Int. Mtg Mast Cell (In the Press).

Denman, A. M., Platts-Mills, T., Brereton, P. J., Doddymead, L., Soan, R., Pelton, B. K., Loewi, G., Shiner, M. \& Peters, D. K. (1976). In Antigen Absorption by the Gut [W. A. Hemmings, editor]. p. 167. Lancaster: MTP Press Lid.

Doeglas, H. M. G. (1975). Br. 7. Derm. 93, 135.

Esteban, M. M., Casas, J. A. O., Borrego, M. T. L. \& Marcos, C. P. (1977). Acta allerg. 32, ${ }^{1} 3$.

Falchuk, K. R. \& Isselbacher, K. J. (1976). Gastroenterology, 70, 5.

Finn, R. \& Cohen, H. N. (1978). Lancet i, 426.

Frier, S. \& Berger, H. (1973). Lancet i, 913.

Galant, S. P., Bullock, J. \& Frick, O. I. (1973). Clin. Allergy 3, 363 .

Hoffman, D. R. \& Haddad, Z. H. (1974). Ұ. Allergy clin. Immunol. 54, 165.

Johnstone, D. E. (1978). Ann. Allergy 40, I 10.

Jukes, T. H. (1977). New Eng. J. Med. $297,427$.

Katz, A. J., Falchuk, Z. M., Strober, W. \& Shwachman, H. (1976). New Eng. J. Med. 295, 131. Klein, N. C., Hargrove, R. L., Sleisenger, M. H. \& Jeffries, G. H. (1970). Medicine 49, 299. Lessof, M. H., Buisseret, P. D., Merrett, T., Wraith, D. G. \& Youlten, L. J. F. (1979). Proc. Int. Mtg Mast Cell (In the Press).

Mackarness, R. (1976). Not All in the Mind, Pan Books.

May, C. D. \& Block, S. A. (1978). Allergy 33, 166.

Paganelli, R., Levinsky, R. J., Brostoff, J. \& Wraith, D. G. (1979). Lancet i, 270.

Platts-Mills, T. A. E. \& Denman, A. M. (1976). F. hum, Nutr. 30, 141 .

Rieger, C. H. L. \& Ruthberg, R. M. (1975). F. Pediat. 87, 5 I5.

Soothill, J. F. (1976). Proc. R. Soc. Med. 69, 439.

Walker, W. A., Wu, M., Isselbacher, K. J. \& Bloch, K. J. (1975). Gastroenterology 69, 1223.

Zanjanian, M. H. (1976). Ann. Allergy 37, 208. 